\title{
An iterative scheme to hierarchically structured optimal energy management of a microgrid
}

\author{
Daniele Ioli, Alessandro Falsone, Maria Prandini
}

\begin{abstract}
In this paper we address the optimal energy management of a microgrid composed of multiple sub-units, each one including one or more buildings sharing some common resources. The goal of the microgrid operator is to match a given electrical energy profile agreed with the operator of the main grid. We propose a decentralized solution scheme based on a hierarchical structure involving three layers: single building, sub-unit, and microgrid operator. At the level of each building, thermal and electrical energy requests are minimized while guaranteeing a certain comfort and quality of service to the building occupants. Optimization of the use of common resources (storages and technological devices) is performed by each sub-unit based on the energy requests of the buildings composing the sub-unit and the cost signal received by the microgrid operator. Each sub-unit minimizes its electrical energy cost as computed based on its own cost signal, while the microgrid operator updates all cost signals based on the outcome of the decentralized optimization, so as to coordinate the sub-units and match the given reference profile. A numerical example shows the efficacy of the approach.
\end{abstract}

\section{INTRODUCTION}

This paper deals with the optimal energy management of a microgrid involving multiple sub-units, each one composed of one or multiple buildings, that are characterized by their own consumption profile in terms of thermal and electrical energy needed to guarantee certain service and comfort levels. Buildings belonging to the same sub-unit share some resources and, possibly, renewable electrical energy sources as well. We suppose that the microgrid is not operating in island mode and, indeed, it can eventually buy/sell electrical energy from/to the main grid when its overall electrical energy balance is not zero.

At the level of each building, thermal and electrical energy requests can be minimized by e.g. slightly varying the temperature set-point. At the sub-unit level, storages and technological devices (e.g. chillers providing cooling energy and Combined Heat and Power (CHP) plants producing heat and electrical energy, smart appliances, etc. [1]) that can be switched on/off and used at different efficiency levels are available, which allows to shift in time the electrical energy request, at least to some extent, see e.g. [2], [3], [4]. The idea is that the microgrid operator can exploit this flexibility in order to minimize the overall electrical energy cost or even try to match a given reference consumption profile agreed with the operator of the main grid over some given time frame. From the main grid perspective, microgrids are like big consumers and knowing ahead of time their electrical

This work is partially supported by the European Commission under the project UnCoVerCPS with grant number 643921.

Daniele Ioli, Alessandro Falsone, and Maria Prandini are with Dipartimento di Elettronica, Informazione e Bioingegneria, Politecnico di Milano, Milano, Italy daniele.ioli, alessandro.falsone, maria.prandini\}@polimi.it energy request profiles can be particularly useful to make a better planning of reserves and ancillary services. As for the microgrid, its advantage is that it can agree on a lower electrical energy price.

In this paper we address the problem of the microgrid matching a given reference profile. A centralized approach where optimization is performed by the microgrid operator may be computationally intensive and requires exchanging a large amount of data that are available locally at the building level. Here we propose a decentralized scheme based on a hierarchical structure to alleviate these difficulties. The hierarchy involves three layers: single building, sub-unit, and microgrid operator. At the level of each building, thermal and electrical energy requests are minimized while guaranteeing a certain comfort and quality of service to the building occupants. Optimization of storages and technological devices functioning is performed by each sub-unit based on the energy requests of the buildings composing the sub-unit and the cost signal received by the microgrid operator. Each subunit minimizes its electrical energy cost as computed based on its own cost signal, while the microgrid operator updates all cost signals based on the outcome of the decentralized optimization, so as to coordinate the sub-units and match the given reference profile. Our work was inspired by [1], where an energy management problem is solved by means of a three-steps iterative method in which a different hierarchical structure is exploited. Following [5], the energy request minimization translates into a linear optimization problem. As in [6], the energy cost minimization performed at the sub-unit level translates into solving a Mixed Integer Linear Program (MILP) using the Mixed Logical Dynamical (MLD) systems framework [7], whereas the iterative update of the cost signals is based on some parameter sensitivity analysis on the underling MILPs. Note that sensitivity analysis of an optimization problem involving discrete optimization variables is difficult since an infinitesimal parameter variation may result in an abrupt change of the solution. Here, we perform it according to some heuristic scheme. We are currently studying a solution based on the formal algorithm proposed in [8], which apparently fits our framework since changes of the cost signals translates into changes of some parameters appearing in the constraints. A numerical example is presented, which shows that the proposed approach appears promising.

\section{PROBLEM DESCRIPTION AND DECENTRALIZED HIERARCHICAL SOLUTION}

Consider a microgrid composed of multiple sub-units, each one containing one or more buildings that share some common resources including thermal storages, and devices such as chillers and CHP plants. Storages allow to shift in 
time the energy requests, whilst devices can be switched on/off and be operated at different working points characterized by a different efficiency so as to save energy. In turn, each building can minimize its thermal energy and electrical energy requests to some extent.

Our goal is to design an optimal control strategy for tuning the consumption at the building level and for handling the common resources at the sub-unit level so that the overall electrical energy profile of the microgrid over some reference time horizon matches a given reference profile that has been agreed with the main grid operator. Dynamics of sub-units and of buildings in the sub-unit are decoupled, the coupling in the problem is brought in by the objective function, since the electrical energy profile of the microgrid is obtained by summing up the sub-units electrical energy requests.

A centralized approach obviously leads to an optimal solution, but typically involves a large number of optimization variables, including binary ones related to the on/off switching of devices. Moreover, its implementation requires that local information related, e.g., to number of occupants, ambient temperature, be transmitted to some central unit running the computations. For these reasons, we opt for a decentralized solution that builds upon the natural decomposition of the problem into three layers: the lowest one, at the building level, the intermediate one, at the sub-unit level, and the highest one, at the microgrid operator level. At the lowest layer we aim at optimizing thermal energy consumption; at the intermediate layer we optimize the use of the shared resources so as to satisfy the energy request of the buildings inside the sub-unit while minimizing the overall energy cost; and at the highest layer, we coordinate all the sub-units so as to match the agreed profile via an appropriate tuning of the cost signals that are used by the intermediate layer to compute the energy cost. The information exchange is then confined to the transmission of the thermal energy request (from the buildings to the sub-unit), the cost signal (from the microgrid operator to the sub-units) and the electrical energy profile (from the sub-units to the microgrid operator).

In the following subsections, we illustrate the solution to the optimization problems posed at each layer, starting from the lowest one, at the building level.

\section{A. Lowest layer: energy optimization at the building level}

At this layer, minimization of the energy request at the level of the building is performed over the reference time horizon. One can adopt in principle any method for optimal energy management. For example, one can adopt the approach in [5] where a convex problem formulation is introduced for the optimal energy management of a single building with a cooling system. The building consists of multiple zones. Instead of taking as control inputs flows and temperatures of the chiller water circuit, that will result in complex nonlinear models [9], [10], the set-point of the temperature in each zone is taken as control variable, with the understanding that a low level controller is making the zone temperature track its set-point.

In order to minimize the cooling energy required for cooling the building over a finite time horizon $\left[t_{i}, t_{f}\right]$, we discretize such a time horizon in $M$ time slot of duration
$\Delta t$, and compute the cost function as

$$
J=\sum_{k=1}^{M} \sum_{j=1}^{n_{z}} E_{c, j}(k),
$$

where $E_{c, j}(k)$ is the energy required to cool down the $j$-th zone during time slot $k$, and $n_{z}$ is the number of zones that compose the building. $E_{c, j}(k)$ is the sum of four contribution, namely

$$
E_{c, j}(k)=E_{w, j}(k)+E_{p, j}(k)+E_{\text {int }, j}(k)+E_{z, j}(k),
$$

where $E_{w, j}(k)$ is the heat transferred to zone $j$ from the adjacent walls, $E_{p, j}(k)$ is the heat generated by people that are occupying zone $j, E_{\text {int }, j}(k)$ is the heat generated by electrical equipment, lighting, and radiation through windows, and $E_{z, j}(k)$ is the energy required to track the set-point in zone $j$.

Let $T_{z, j}(k)$ be the temperature set-point value of zone $j$ at the end of the $k$-th time slot. In [5], it is shown that if we set $\boldsymbol{E}_{\boldsymbol{c}}=\left[E_{c, 1}(1) \cdots E_{c, n_{z}}(1) \cdots E_{c, 1}(M) \cdots E_{c, n_{z}}(M)\right]^{\top}$, then

$$
\boldsymbol{E}_{\boldsymbol{c}}=A \boldsymbol{T}_{\boldsymbol{z}}+B,
$$

where $\boldsymbol{T}_{\boldsymbol{z}}=\left[T_{z, 1}(1) \cdots T_{z, n_{z}}(1) \cdots T_{z, 1}(M) \cdots T_{z, n_{z}}(M)\right]^{\top}$ is the control input vector, $A$ is a suitable matrix, and $B$ is a properly defined vector. Both $A$ and $B$ depend on the structure of the building and its thermal characteristics, on the occupancy profile of each zone, and on the external disturbances such as outside temperature, shortwave and long wave radiation, and the initial temperature of each zone, (see [5] for details).

Finally, the building optimization problem is given by

$$
\begin{aligned}
\underset{\boldsymbol{T}_{\boldsymbol{z}}}{\min } & J \\
\text { subject to: } & \boldsymbol{T}_{\boldsymbol{z}, \min } \leq \boldsymbol{T}_{\boldsymbol{z}} \leq \boldsymbol{T}_{\boldsymbol{z}, \max } \\
& \boldsymbol{E}_{\boldsymbol{c}} \geq 0,
\end{aligned}
$$

where $\boldsymbol{T}_{\boldsymbol{z}, \min }$ and $\boldsymbol{T}_{\boldsymbol{z}, \max }$ are the lower and upper comfort limits for the zone temperatures. Note that, differently from the work in [5], we do not consider neither the chiller, nor the thermal storage, whose optimization is moved to the upper layer.

\section{B. Intermediate layer: common resources optimization at the sub-unit level}

We consider the case when each sub-unit contains multiple buildings that share some common resources. Our goal is to optimize the use of such resources so as to minimize the cost of the consumed energy while matching the energy request of each building in the sub-unit.

Suppose there are $n_{b}(i)$ buildings in a given sub-unit $i$. Let $E_{r, c}^{(j)}(k)$ denote the cooling energy requested from building $j$ during time slot $k, E_{r, h}^{(j)}(k)$ the heating energy request (e.g. hot water), and $E_{r, \ell}^{(j)}(k)$ the electrical energy request (obtained as the difference between the electrical energy needed and the amount produced by renewable sources). Then, at the sub-unit level we shall optimize the cost of providing $E_{r, c}(k)=\sum_{j=1}^{n_{b}(i)} E_{r, c}^{(j)}(k), E_{r, h}(k)=$ $\sum_{j=1}^{n_{b}(i)} E_{r, h}^{(j)}(k), E_{r, \ell}(k)=\sum_{j=1}^{n_{b}(i)} E_{r, \ell}^{(j)}(k), k=1, \ldots, M$ along the time horizon $\left[t_{i}, t_{f}\right]$. To this purpose we need 


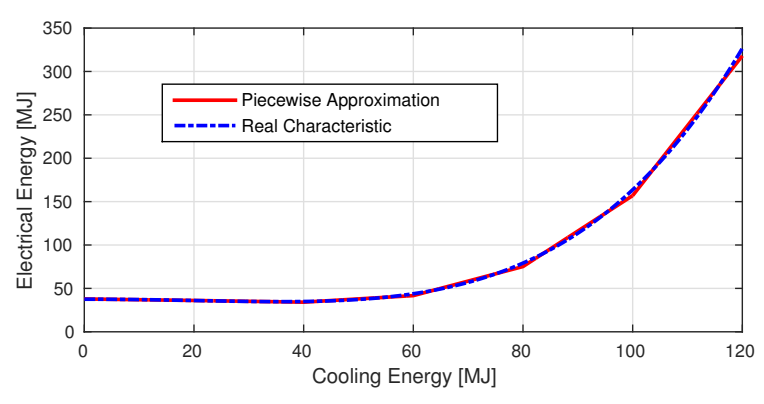

Fig. 1. Chiller nonlinear characteristic and piecewise affine approximation.

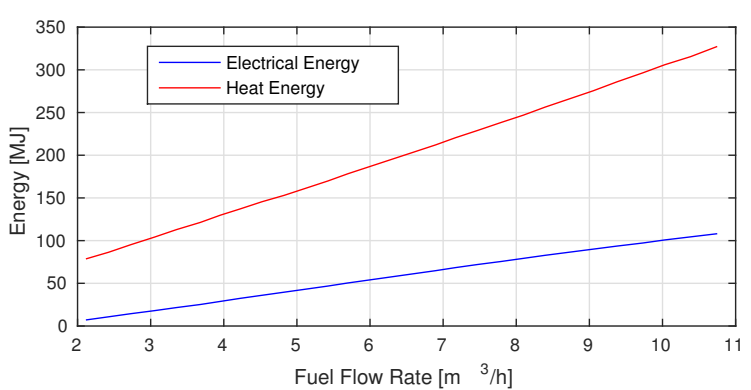

Fig. 2. Microturbine static characteristics. to introduce appropriate models for the common resources that are available in the sub-unit and define a suitable cost function.

We next focus, in particular, on the case of a sub-unit where the common resources consist of a chiller plant with a thermal storage for the cold water, and a CHP plant composed of a microturbine which burns gas to produce electrical energy and, as a side product, thermal power which is used for water heating and stored in a thermal storage for hot water.

As for the chiller, we use a piecewise affine approximation (see Figure 1) of the widely used nonlinear static $\mathrm{Ng}$ Gordon model, [11], providing the electrical energy $E_{c h, \ell}$ as a function of the cooling energy $E_{c h, c}$. As can be seen from Figure 1, even though the cooling energy requested to the chiller is low, a relevant amount of electrical energy is drawn. Since we are interested in minimizing the cost of the energy consumption, we introduce the possibility of turning the chiller off. As a result, the optimization variables for the chiller unit are the cooling energy request $u_{c h}(k)$ for each time slot $k=1, \ldots, M$, and a binary variable $\delta_{c h}(k)$ that represents the on/off status of the chiller, with $k=1, \ldots, M$. Precisely, $\delta_{c h}(k)=1$ if the chiller is on, and $\delta_{c h}(k)=0$ otherwise. Denoting by $\eta_{c h}(\cdot)$ the piecewise affine characteristic of the chiller, the electrical energy $E_{c h, \ell}(k)$ can be computed as $E_{c h, \ell}(k)=\delta_{c h}(k) \eta_{c h}\left(u_{c h}(k)\right)$.

A similar discussion holds for the CHP unit. We consider here a microturbine. Assuming that the microturbine has it own low level controller, we can model the machine with two static characteristics: electrical power production and heat production, both as a function of the fuel volumetric flow rate. In particular, in Figure 2, we can see that both curves are almost linear, the one for the heat production being higher than the other one. The microturbine specifications require a minimum fuel volumetric flow rate $u_{m t}^{\min }$ for the unit to be operative. In order to minimize the gas consumption, and thus the costs, we introduce also in this case the possibility to turn the unit on and off. In [12], a power plant turbine is said to have a transient from off to on, so we assumed that the duration of this transient is lower than the time slot $\Delta t$. For our control purposes we take the fuel volumetric flow rate $u_{m t}(k)$, and a binary variable $\delta_{m t}(k)$ that represents the state of the microturbine, for each time slot $k=1, \ldots, M$ as our control variables. If $\delta_{m t}(k)=1$, then the microturbine is on, $\delta_{m t}(k)=0$ otherwise.

The electrical energy $E_{m t, \ell}(k)$ and the heat $E_{m t, h}(k)$ produced by the microturbine during the time slot $k$ are expressed as affine functions of the fuel volumetric flow rate $u_{m t}(k)$, that is supposed to be constant in each time slot, namely $E_{m t, \ell}(k)=\delta_{m t}(k)\left(m_{\ell} u_{m t}(k)+q_{\ell}\right), E_{m t, h}(k)=$ $\delta_{m t}(k)\left(m_{h} u_{m t}(k)+q_{h}\right)$, where $m_{\ell}, q_{\ell}, m_{h}$, and $q_{h}$ are positive coefficients suitably defined so as the static characteristics of the microturbine match the ones in Figure 2.

Each sub-unit can benefit from the presence of two thermal storages, a cold one and a hot one. As in [5], we model the storages as first order systems $S_{c}(k+1)=a_{c} S_{c}(k)-s_{c}(k)$, $S_{h}(k+1)=a_{h} S_{h}(k)-s_{h}(k)$, where $s_{c}(k)$ and $s_{h}(k)$ are control variables that represents the amount of energy drawn from the storages (cold and hot storage respectively) during time slot $k$, and $a_{c}, a_{h} \in(0,1)$ are coefficient introduced to model energy losses.

The cost function to be minimized at the sub-unit level is the sum of the operational costs along the time horizon $\left[t_{i}, t_{f}\right]$. The costs in each time slot $k$ are mainly constituted of two contribution: the cost $C_{\ell}(k)$ of the electrical energy consumed by the chiller to produce cooling energy and the cost $C_{f}(k)$ of the fuel burned by the CHP plant. In order to avoid continuous (and unrealistic) switchings of both the chiller and the microturbine, we also introduce some startup costs $C_{c h}^{\xi}$ and $C_{m t}^{k}$, respectively, and introduce additional cost contributions per time slot as follows:

$$
\begin{aligned}
C_{c h}(k) & =C_{c h}^{\xi} \cdot \max \left\{\delta_{c h}(k)-\delta_{c h}(k-1), 0\right\} \\
C_{m t}(k) & =C_{m t}^{\xi} \cdot \max \left\{\delta_{m t}(k)-\delta_{m t}(k-1), 0\right\}
\end{aligned}
$$

The fuel costs are proportional to the amount of fuel consumption during the $k$-th time slot, formally

$$
C_{f}(k)=\psi_{f} \cdot \delta_{m t}(k) \cdot u_{m t}(k) \cdot \Delta t,
$$

where $\psi_{f}$ is the unitary cost of the fuel.

Finally, the electrical energy cost $C_{\ell}(k)$ is a piecewise affine function of the electrical energy

$$
E_{\ell}(k)=E_{c h, \ell}(k)+E_{r, \ell}(k)-E_{m t, \ell}(k),
$$

which is positive if the required energy is more than the produced energy, and negative otherwise. The cost signal $C_{\ell}(\cdot)$ is parameterized through the vector of parameters $\psi(k)=\left[\begin{array}{llll}\psi_{\min }(k) & \psi_{\text {sell }}(k) & \psi_{\text {buy }}(k) & \psi_{\max }(k)\end{array}\right]^{\top}$, where $\psi_{\min }(k) \in(-\infty, 0)$ and $\psi_{\max }(k) \in(0,+\infty)$ are the maximum amount of energy that can be sold/bought to/from the microgrid, $\psi_{\text {buy }}(k) \in(0,+\infty)$ is the unitary cost of the electrical energy, and $\psi_{\text {sell }}(k) \in\left(0, \psi_{\text {buy }}(k)\right)$ is the unitary revenue for selling electrical energy. All these quantities are time-varying, but supposed to be constant inside the $k$-th 


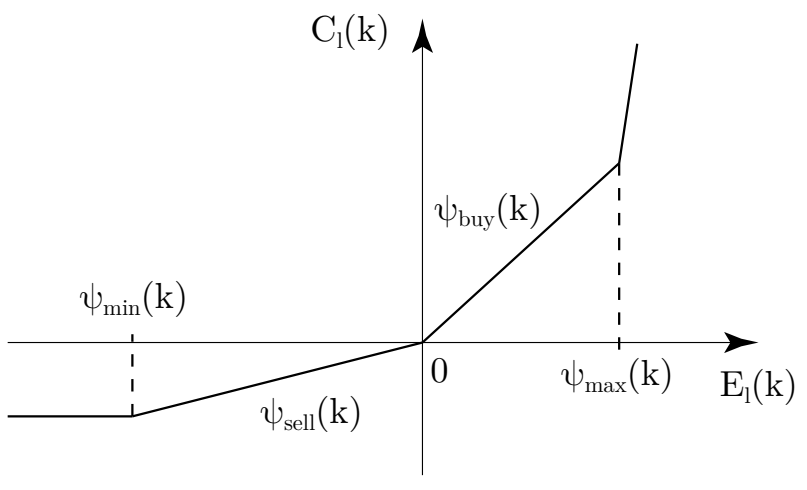

Fig. 3. Electrical energy cost signal at time slot $k$.

time slot. Function $C_{\ell}(k)$ is represented in Figure 3 as a function of $E_{\ell}$, with $\psi_{\text {buy }}(k)$ and $\psi_{\text {sell }}(k)$ denoting the slopes of the curves. Hard constraints on the maximum energy that can be bought/sold are relaxed and coded in $C_{\ell}(k)$ through a unitary cost of buying energy that is much larger than $\psi_{\text {buy }}(k)$ if $E_{\ell}(k)$ exceeds $\psi_{\max }(k)$, and a unitary cost of selling energy that is zero if the amount of energy to be sold exceeds $\left|\psi_{\min }(k)\right|$.

Note that $C_{\ell}(k)$ has to be interpreted not as a real cost but a cost signal used by the microgrid operator to make the microgrid energy profile match the one agreed with the main grid operator through an appropriate tuning of the parameter vector $\psi(k), k=1, \ldots, M$.

Finally, collecting all optimization variables in vector

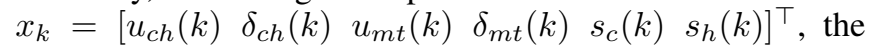
optimization problem that needs to be solved is given by:

$$
\min _{\left\{x_{k}\right\}_{k=1}^{M}} \sum_{k=1}^{M}\left(C_{\ell}(k)+C_{f}(k)+C_{c h}(k)+C_{m t}(k)\right)
$$

subject to:

$$
\begin{aligned}
& \delta_{c h}(k), \delta_{m t}(k) \in\{0,1\} \\
& 0 \leq u_{c h}(k) \leq u_{c h}^{\max }, u_{m t}^{\min } \leq u_{m t}(k) \leq u_{m t}^{\max } \\
& E_{r, c}(k)=\delta_{c h}(k) u_{c h}(k)+s_{c}(k) \\
& E_{r, h}(k)=E_{m t, h}(k)+s_{h}(k) \\
& E_{\ell}(k)=E_{c h, \ell}(k)+E_{r, \ell}(k)-E_{m t, \ell}(k) \\
& S_{c}(k+1)=a_{c} S_{c}(k)-s_{c}(k) \\
& S_{h}(k+1)=a_{h} S_{h}(k)-s_{h}(k) \\
& 0 \leq S_{c}(k) \leq S_{c}^{\max }, 0 \leq S_{h}(k) \leq S_{h}^{\max } \\
& k=1, \ldots, M
\end{aligned}
$$

where $u_{c h}^{\max }$ is the maximum amount of energy that can be requested to the chiller, $u_{m t}^{\max }$ is the maximum value for the volumetric flow rate of the microturbine, and $S_{c}^{\max }$ and $S_{h}^{\max }$ are the maximum capacities of the cold and hot storages respectively. Problem (2) is a MILP posed on a system that can described according to the MLD formalism, [7]. Details are omitted here.

\section{Highest layer: reference profile tracking at the microgrid operator level}

At this layer the goal is coordinating all the sub-units so as to match some electrical energy profile.
Suppose that the microgrid is composed of $n_{s}$ sub-units. Let $\tilde{x}^{i}=\left[\tilde{x}_{1}^{i} \cdots \tilde{x}_{M}^{i}\right]^{\top}$ be the optimal solution to (2) for the $i$-th sub-unit for given energy profiles $E_{r, c}^{i}(k)$ and $E_{r, h}^{i}(k)$, $k=1, \ldots, M$, with $i=1, \ldots, n_{s}$. The solution depends on the vector of parameters $\psi^{i}(k), k=1, \ldots, M$, defining the electrical energy cost signal $C_{\ell}^{i}(k), k=1, \ldots, M$, for the $i$ th sub-unit. Let us denote with $E_{\ell}^{i}=\left[E_{\ell}^{i}(1) \cdots E_{\ell}^{i}(M)\right]^{\top}$ the electrical energy profile for the $i$-th sub-unit corresponding to $\tilde{x}^{i}$. Also $E_{\ell}^{i}$ depends on $\psi^{i}(k), k=1, \ldots, M$, and we shall make this dependence explicit via the notation $E_{\ell}^{i}\left(\psi^{i}(1), \ldots, \psi^{i}(k), \ldots, \psi^{i}(M)\right)$.

Suppose to perform a small perturbation $\delta \psi_{p}^{i}(k)$ from the current value $\bar{\psi}_{p}^{i}(k)$ of the parameter $\psi_{p}^{i}(k)$, with $p \in P=$ $\{\min$, sell, buy, $\max \}$. One can then estimate the effects of such variation on the energy profile using

$$
\begin{aligned}
\nabla_{p, k}^{i}= & E_{\ell}^{i}\left(\bar{\psi}_{\min }^{i}(1), \ldots, \bar{\psi}_{p}^{i}(k)+\delta \psi_{p}^{i}(k), \ldots, \bar{\psi}_{\max }^{i}(M)\right) \\
& -E_{\ell}^{i}\left(\bar{\psi}_{\min }^{i}(1), \ldots, \bar{\psi}_{p}^{i}(k), \ldots, \bar{\psi}_{\max }^{i}(M)\right)
\end{aligned}
$$

with $p \in P$.

In order to make the microgrid electrical energy profile match at best the one agreed with the main grid operator, say $\bar{E}_{k}, k=1, \ldots, M$, we minimize $\mathcal{D}=$ $\sum_{k=1}^{M}\left|\sum_{i=1}^{n_{s}} E_{\ell}^{i}(k)-\bar{E}_{k}\right|$, where we recall that $E_{\ell}^{i}$ is a function of $\psi^{i}(1), \ldots, \psi^{i}(M), i=1, \ldots, n_{s}$.

We can approximately solve this problem by allowing, for each sub-unit $i$, a variation $\gamma_{p, k}^{i}$ of one single parameter $\psi_{p}^{i}(k)$, using $\nabla_{p, k}^{i}$ to estimate the impact on $\mathcal{D}$. The variation $\gamma_{p, k}^{i}$ must be smaller than $\delta \psi_{p}^{i}(k)$ in amplitude. To ensure that at most one parameter $\psi_{p}^{i}(k)$ is changed for each subunit $i$, we define a set of binary variables $b_{p, k}^{i} \in\{0,1\}$, $\forall k, i, p$, that must satisfy $\sum_{k=1}^{M} \sum_{p \in P} b_{p, k}^{i} \leq 1$.

To choose the optimal values for $\gamma_{p, k}^{i}$ we solve the following optimization program

$$
\min _{\left\{\gamma_{p, k}^{i}, b_{p, k}^{i}\right\}_{p \in P, k=1, \ldots, M, i=1, \ldots, n_{s}}} \tilde{\mathcal{D}}
$$

subject to:

$$
\begin{aligned}
& b_{p, k}^{i} \in\{0,1\}, \sum_{k=1}^{M} \sum_{p \in P} b_{p, k}^{i} \leq 1 \\
& \left|\gamma_{p, k}^{i}\right| \leq \delta \psi_{p}^{i}(k), b_{p, k}^{i}=0 \Longleftrightarrow \gamma_{p, k}^{i}=0 \\
& p \in P, k=1, \ldots, M, i=1, \ldots, n_{s}
\end{aligned}
$$

where the cost function $\tilde{\mathcal{D}}$ is defined as

$$
\tilde{\mathcal{D}}=\sum_{k=1}^{M}\left|\sum_{i=1}^{n_{s}}\left(E_{\ell}^{i}(k)+\sum_{p \in P}\left(b_{p, k}^{i} \nabla_{p, k}^{i} \gamma_{p, k}^{i}\right)\right)-\bar{E}_{k}\right| .
$$

\section{Iterative scheme}

The iterative scheme is summarized in Algorithm 1.

Note that steps 1 to 6 , and 9 to 14 , that are the ones with the highest computational burden, can all be performed in parallel in each sub-unit. The only centralized problem that is solved is the lightweight optimization problem in step 15 and the simple update step 16.

\section{NUMERICAL EXAMPLE}

To show the effectiveness of the proposed approach we consider a microgrid composed of three sub-units with given 
cooling and heating energy requests as depicted in Figure 4. The electrical energy requests of the three sub-units are reported in Figure 5. The reference time horizon is a day discretized in $M=24$ time slots of 1 hour. These profiles should be thought of as generated by step 1 of Algorithm 1 . We next focus on the decentralized solution for the sub-unit coordination problem.

In Algorithm 1, $E_{r, c}^{i}(k)$ and $E_{r, h}^{i}(k)$ are then the ones in Figure 4, and $E_{r, \ell}^{i}(k)$ that in Figure 5. As for $\psi_{0}(k)$, $\psi_{\text {buy }, 0}(k)$ and $\psi_{\text {sell, } 0}(k)$, they are set according to Figure 6 , while $\psi_{\max , 0}(k)=30 \mathrm{MJ}$, and $\psi_{\min , 0}(k)=-30 \mathrm{MJ}$, for $k=1, \ldots, M$.

At iteration 1 each sub-unit solves problem (2) with the (same) initial cost $\psi_{0}(k)$. Figure 7 plots the resulting electrical energy profile of sub-unit 1 . As we can clearly see by comparing Figure 6 and Figure 7, when the electrical energy cost is low the sub-unit opts for buying the energy from the main grid rather than producing it with the microturbine. On the other hand, between 8AM and 1PM, when the energy cost is high, the sub-unit is producing energy to be autonomous, even though the thermal heating energy produced as a side effect by the microturbine is not needed (see the heating energy request top profile in Figure 4).

Another important aspect is that the introduced possibility to turn the chiller on and off is indeed exploited, despite its additional costs, and indeed the sub-unit either operates the chiller at its best efficiency point, or turns it off. This is shown in Figure 8, where the Coefficient Of Performance (COP) of the chiller and its actual working point (red dot) are reported. Most of the time the chiller works at the maximum of its COP, only a few times it works in nonoptimal conditions, and it is turned off 4 times.

Steps from 7 to 16 of Algorithm 1 aim at solving the

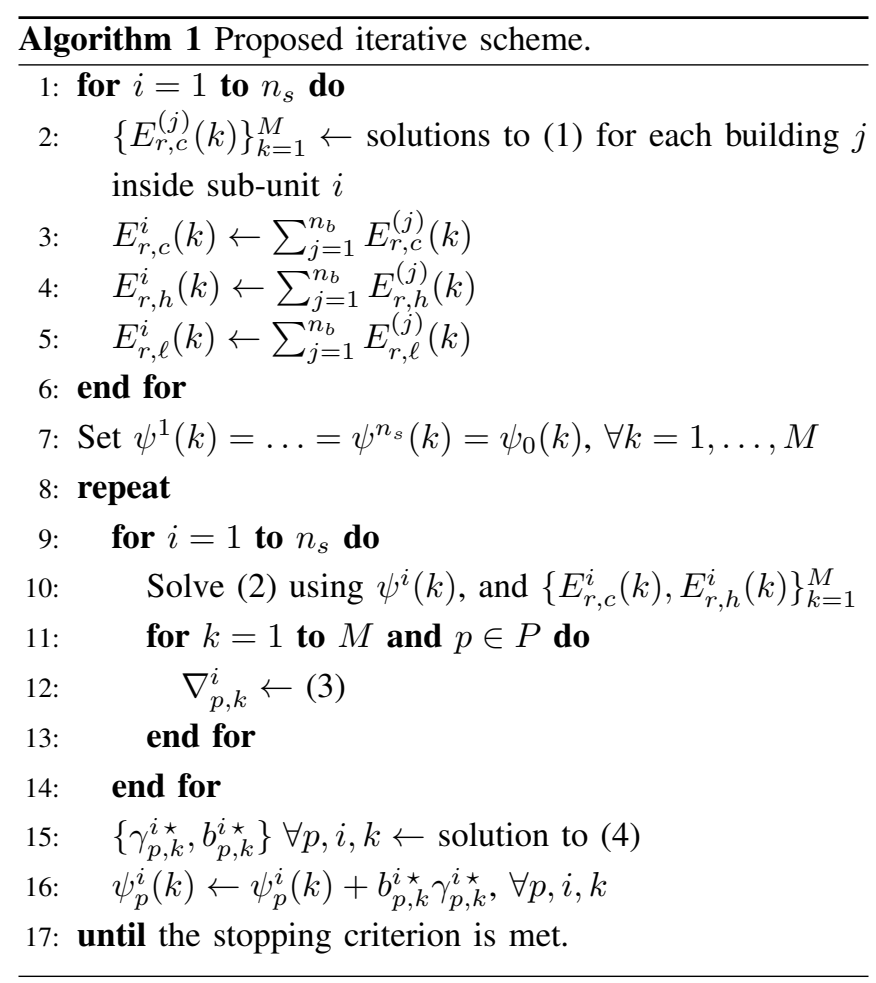

sub-unit coordination problem and are run until convergence, with $\bar{E}_{k}=0, k=1, \ldots, M$, meaning that the desired electrical energy profile for the microgrid corresponds to demand being equal to generation. At each iteration, the estimated value $\tilde{\mathcal{D}}$ of the cost function is computed using (3) in (5) and solving (4). The evolution of $\tilde{\mathcal{D}}$ and the actual $\operatorname{cost} \mathcal{D}$ is reported in Figure 9 . The cost $\mathcal{D}$ is decreasing, not monotonically though. In most iterations the two values are the same, but in iterations 3,5 , and $7, \mathcal{D}$ of the cost function is higher than the predicted one. This happens because the optimization problem (2) involves binary variables, hence if the performed variation on the parameters is different from the one used to compute (3), then it might happen that in the new solution a binary variable has changed its value, thus producing an unpredicted change of the actual cost. This can be avoided by allowing only parameters variations for which the impact on the cost function has been previously computed. In Figure 9 we also report the optimal value that can be obtained solving the centralized problem. As can be seen, the proposed solution approaches the optimal value without actually reaching it. The implementation of a dynamic step-size in the computation of (3) and in the update step 15 of the algorithm should allow to actually converge to the global optimum.

Finally, Figure 10 shows the electrical energy profiles of each sub-unit that are obtained by solving (2) at the 1st iteration and at the 15-th iteration. As can be seen by comparing the orange bars with the green ones, the algorithm mainly acts on the time slots from 9 to 12 trying to match the reference energy profile, and thus trying to redistribute the exceeding electrical energy from sub-unit 3 to the other two sub-units (see Figure 5 for the electrical energy request of each sub-unit). In the other time slots, when all three subunits need electrical energy, the algorithm tries to minimize (whenever possible) the total energy request, see e.g. time slots 1,2 , and 19 to 23 , in which the energy request is greatly reduced at iteration 15 in comparison with iteration 1 .

\section{CONCLUSIONS}

In this paper we proposed a decentralized hierarchical structured solution to the optimal energy management of a microgrid. The approach was tested on a numerical example and looks promising. Still, much work needs to be done

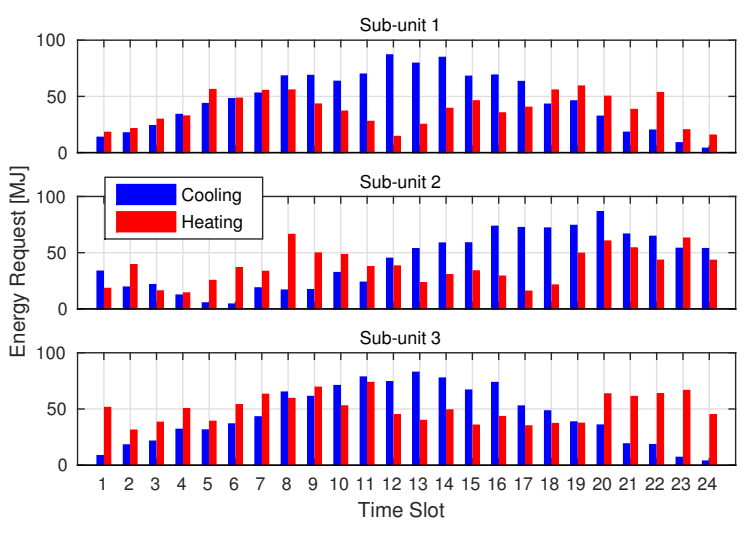

Fig. 4. Cooling and heating energy request for the three sub-units. 


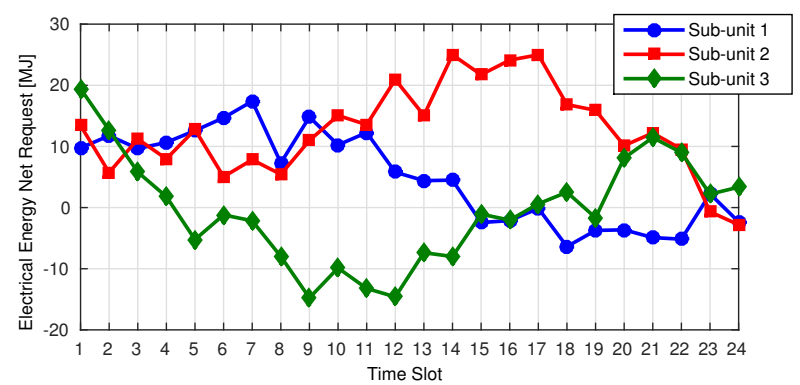

Fig. 5. Electrical energy request (required energy minus produced by renewable sources) for the three sub-units.

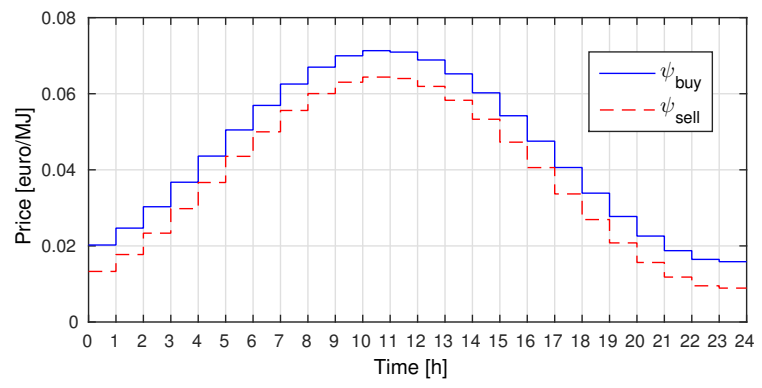

Fig. 6. Initial profiles $\psi_{\text {buy }, 0}(k)$ and $\psi_{\text {sell }, 0}$.

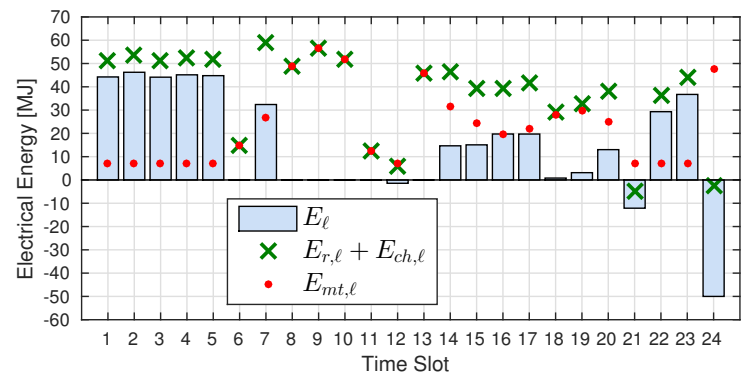

Fig. 7. Sub-unit 1 optimal electrical energy profile after the first iteration.

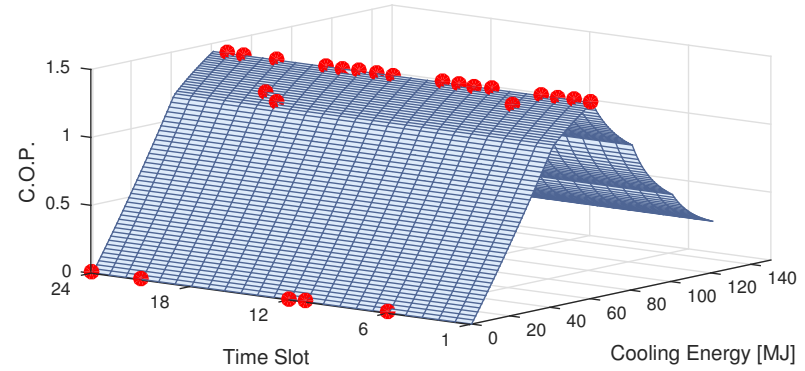

Fig. 8. Sub-unit 1: chiller working points and COP curve.

to make it applicable to a real context, where uncertainty affects the demand and also the generation side because of the stochastic nature of renewable sources. A possibility is to rephrase the approach in a robust context, first confining the uncertainty to a set of realizations with a priori-defined (high) probability $1-\varepsilon$ as suggested in e.g. [9].

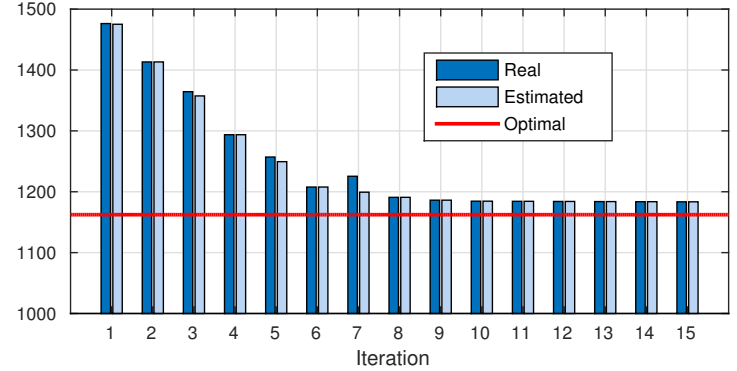

Fig. 9. Expected and actual cost function through iterations.

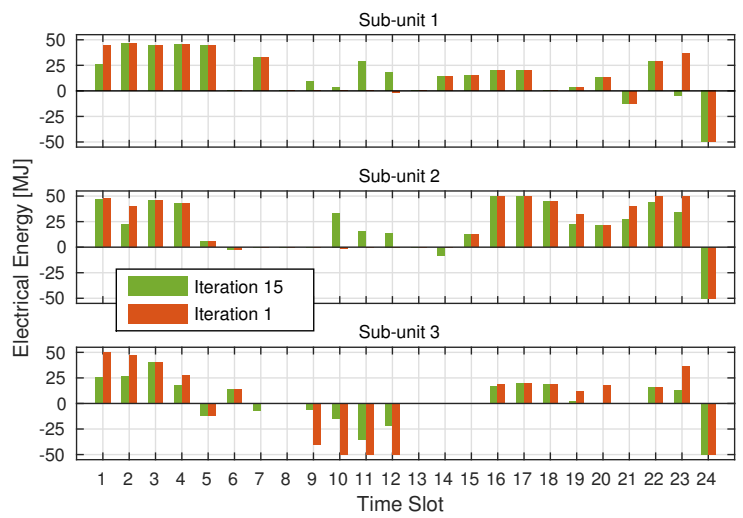

Fig. 10. Electrical energy profiles: 1-st and 15-th iterations.

\section{REFERENCES}

[1] A. Molderink, On the three-step control methodology for smart Grids. CTIT Ph.D thesis Series No, 11-196, University of Twente, The Netherlands, 2011.

[2] K. Deng, Y. Sun, A. Chakraborty, Y. Lu, J. Brouwer, and P. G. Mehta, "Optimal scheduling of chiller plant with thermal energy storage using mixed integer linear programming," in $A C C$, Washington, DC, USA, June 17-19 2013

[3] K. M. Powell, W. J. Cole, U. F. Ekariaka, and T. F. Edgar, "Dynamic optimization of a campus cooling system with thermal storage," in ECC, Zurich, Switzerland, July 17-19 2013, pp. 4077-4082.

[4] Y. Ma, F. Borrelli, B. Hencey, A. Packard, and S. Bortoff, "Model predictive control of thermal energy storage in building cooling systems," in CDC/ECC, Dec 2009, pp. 392-397.

[5] D. Ioli, A. Falsone, and M. Prandini, "Optimal energy management of a building cooling system with thermal storage: A convex formulation," in ADCHEM, Whistler, British Columbia, Canada, June 2015.

[6] S. H. Haikarainen C., Pattersson F., "An MILP model for distributed energy system optimization," in Chemical Engeneering Transactions, Dec 2013, pp. 295-300.

[7] A. Bemporad and M. Morari, "Control of systems integrating logic, dynamics, and constraints," Automatica, vol. 35, no. 3, pp. 407-427, 1999.

[8] M. W. Dawande and J. N. Hooker, "Inference-based sensitivity analysis for mixed integer/linear programming," Operations Research, vol. 48, 1998.

[9] F. Borghesan, R. Vignali, L. Piroddi, and M.Prandini, "Approximate dynamic programming-based control of a building cooling system with thermal storage," in IEEE ISGT 2013, Copenhagen, Denmark, October 2013.

[10] N. Ceriani, R. Vignali, L. Piroddi, and M. Prandini, "An approximate dynamic programming approach to the energy management of a building cooling system," in ECC, Zurich, Switzerland, July 2013

[11] M. Gordon and K. Ng, Cool thermodynamics. Cambridge International Science Publishing, 2000.

[12] G. Ferrari-Trecate, E. Gallestey, P. Letizia, M. Spedicato, M. Morari, and M. Antoine, "Modeling and Control of Co-generation Power Plants: A Hybrid System Approach," in International Workshop on Hybrid Systems: Computation and Control, Stanford, California, USA, Mar. 2002, pp. 208-224. 